\title{
TRANSGENIC MOSQUITOES FIGHT AGAINST MALARIA: A REVIEW
}

\author{
Amit Kumar Shrivastava ${ }^{1,3}$, Laxmi Shrestha ${ }^{1}$, Shraddha Prakash ${ }^{2}$, Roshan Kumar Mehta $^{3}$
}

\begin{abstract}
Malaria control with transgenic mosquitoes will be challenging; however, recent advances suggest that it may be a possibility in the foreseeable future. Progress towards discovering refractory genes for rodent malaria and gene drive systems for Drosophila provide hope that similar advances may be made for human malaria in mosquito vector species. That said, the African malaria burden has proved exceptionally difficult to diminish by all means tried thus far; and it is unlikely that transgenic mosquitoes will provide an all-in-one solution. Transgenic mosquitoes should be considered within the context of an integrated vector management strategy which should also include insecticide-treated bed-nets, indoor residual spraying with insecticides and treatment of infected individuals with antimalarial drugs. Integrated strategies will be a necessity for any successful african malaria control program and transgenic mosquitoes should be considered as a potential ingredient in the future goal of continent-wide disease control.
\end{abstract}

KEY WORDS Malaria, transgenic, mosquitoes

1. Department of Pharmacology, Universal College of Medical Sciences, Bhairahawa, Nepal

2. MRD Life Sciences, Lucknow, Uttar Pradesh

3. UCMS College of Pharmacy, Bhairahawa, Nepal

DOI: https://doi.org/10.3126/jucms.v7i1.24695

For Correspondence

Amit Kumar Shrivastava

Department of Pharmacology

Universal College of Medical Sciences

Bhairahawa, Rupendahi, Nepal

Email: sr.akshri@gmail.com 


\section{INTRODUCTION}

Malaria is a mosquito-borne infectious disease of humans and other animals caused by parasitic protozoans (a type of unicellular microorganism) of the genus Plasmodium. Commonly, the disease is transmitted via a bite from an infected female anopheles mosquito, which introduces the organisms from its saliva into a person's circulatory system. In the blood, the protists travel to the liver to mature and reproduce. Malaria causes symptoms that typically include fever and headache, which in severe cases can progress to coma or death. The disease is widespread in tropical and subtropical regions in a broad band around the equator, including much of Sub-Saharan Africa, Asia, and the Americas. The species of Plasmodium can infect and be transmitted by humans. The vast majority of deaths are caused by $P$. falciparum and $P$. vivax, while $P$. ovale and $P$. malariae cause a generally milder form of malaria that is rarely fatal. Malaria is prevalent in tropical and subtropical regions because rainfall, warm temperature, and stagnant water provide habitats ideal for mosquito larvae.

Malaria kills more than a million people each year, primarily children under the age of six. To combat malaria health agencies try to get rid of mosquitoes, which transmit diseases. But a scarcity of resources hampers most control programs, and the insects are increasingly resistant to pesticides after decades of patchwork spraying. The disease organisms are evolving. The single-celled microbes that cause malaria are becoming resistant to widely used, inexpensive anti-malarial drugs such as chloroquine, which have been the first-choice treatment for malaria. ${ }^{2}$ Many research teams are trying to develop vaccines for these diseases, but the complex biology of the two parasites malaria is caused by four species of the protist Plasmodium makes it difficult to predict whether vaccination will eventually confer broad immunity, and although pesticide treated bed nets offer a promising low tech means of preventing bites from malarial mosquitoes at night. ${ }^{3}$

To oppose these grim realities, several research teams (including a group at North Carolina State University) are now exploring a different approach to controlling the spread of mosquito borne diseases, one that would reduce an insect's ability to transmit disease or would induce a population crash among selected disease carrying species. By creating genetic changes in wild mosquitoes. Biologists have already extinguished other insect pests with genetic methods and in the laboratory have blocked the transmission of malaria in mosquitoes with engineered fragments of DNA. ${ }^{4}$ If scientists could breed some of those same genes into the wild mosquito population, the insect's bite might still be a nuisance but it would no longer be a threat. Setting aside concerns over the release of genetically modified mosquitoes (more on that later), there is reason to be optimistic that this strategy might work. ${ }^{5,6}$ Thanks to the tools of molecular biology, geneticists can make very specific changes in mosquito genomes.

In 2002, Case Western Reserve University, modified Anopheles stephensi mosquitoes with genes that blocked the development of Plasmodium berghei, a relative of the malaria parasites that infect human beings. Other scientists have developed genetic strategies that simply kill the mosquitoes that carry a specific engineered gene. But having mosquitoes that don't transmit pathogens in the laboratory doesn't help people in the wider world. ${ }^{4,7}$ Even if scientists bred and released thousands of these transgenic mosquitoes, they wouldn't have much effect on public health unless the genetically altered insects competed with (and eventually replaced) the local strains. Now for the first time, University of Arizona entomologists have succeeded in genetically altering mosquitoes in a way that renders them completely immune to the parasite, a single celled organism called Plasmodium. Someday researchers hope to replace wild mosquitoes with lab bred populations unable to act as vectors, i.e. transmit the malaria-causing parasite. ${ }^{3,8}$ If you want to effectively stop the spreading of the malaria parasite, you need mosquitoes that are no less than 100 percent resistant to it. The researchers targeted one of the many biochemical pathways inside the mosquito's cells. Specifically, they engineered a piece of genetic code acting as a molecular switch in the complex control of metabolic functions inside the cell. The genetic construct acts like a switch that is always set to "on," leading to the permanent activity of a signaling enzyme called Akt. Akt functions as a messenger molecule in several metabolic functions, including larval development, immune response and lifespan.

For the development of transgenic mosquitoes suitable vector transformation systems, a DNA construct having a reporter gene and pathogen effector molecule which is driven by a strong promoter are required. The vector transformation systems used to produce transgenic mosquitoes include virus transducing systems, transposable elements, and endosymbionts. The virus transducing systems include double sub genomic Sindibs virus (SIN) expression vector TE/3'J and SIN replicon. With the help of these viruses transducing systems such as anopheles as and culex mosquitoes could be transformed. Even though virus transducing systems were capable of transforming and integrating transgenes into mosquito genome, they were not capable of spreading the transgene efficiently to mosquitoes because the transgene was inherited to the next generation via Mendelian inheritance only. For the efficient transgene spreading in the natural population, an enormous numbers of mosquitoes were 
required. In order to product such numbers of TMs, transposable elements (TE) and endosymbionts that were capable of spreading the transgene in a non-Mendelian manner was begun to use.

In the past, insecticide resistance genes and antibiotic resistance genes were used as reporter genes when TMs were developed but currently, fluorescent markers such as enhanced green fluorescent protein (EGFP) and Discosoma sp. Red fluorescent protein (dsRED) are used to select transgenic mosquitoes from non-transgenic mosquitoes. ${ }^{10,11}$ Using fluorescent markers as reporter genes saved the time and effort of rearing nontransgenic mosquitoes to adults because the fluorescent protein expressing mosquitoes could be observed and separated even in early larval stages, however, if insecticide resistance genes were used asreporter genes both transgenic and non-transgenic larvae had to be reared until adult stage. ${ }^{12,}{ }^{13}$ Some of the pathogen effector molecules used when producing transgenic mosquitoes were antiparasitic peptides, antimicrobial peptides, antisense RNA, and shRNA. ${ }^{14}$

The World Health Organization (WHO) reports 135-287 millions of malaria cases annually indicating urgent need for new control strategies. ${ }^{15}$ TMs as one of the promising control strategies, the mosquito having a foreign DNA that codes for a synthetic gene, AgCP [SM1] containing CP signal sequence, and coding sequences of four subunits of SM1 under the regulation of $\mathrm{AgCP}$ promoter was constructed and inserted into pBac transposable element (TE) containing vector with enhanced green fluorescent protein (EGFP) gene under the regulation of mosquito eye specific $3 \mathrm{xP} 3$ promoter. ${ }^{16}$ The final DNA construct was then transformed along with transposase enzyme coding helper plasmid into embryos of An. stephensi and EGFP expression in the mosquito eyes was then used for the identification of TMs. ${ }^{17,18}$ The induction of $\mathrm{AgCP}$ promoter of the construct to express SM1 tetramer protein is generally taking place for approximately 24 hours following ingestion of blood meals by the mosquitoes, An. stephensi and CP signal peptide of this protein direct SM1 to be secreted into the midgut to cover its luminal surface to inhibit the invasion of parasite Plasmodium to the epithelium of midgut, which happen at the same time period of SM1 protein expression resulting in fewer parasite invasions to salivary glands making less parasites in the duct lumen for transmission. The TMs developed in this manner will pass on the construct to future generations replacing the wild type mosquitoes. Therefore, the transmission of the malaria parasite can be curtailed significantly. However, the emergence of resistant parasite through natural selection due to genetic diversity and mutability of Plasmodium has been the issue of concern. As a solution for this, the modification of mosquito genomes with multiple genes that could inhibit the parasite development by different mechanisms were carried out resulting mosquitoes called transgenic hybrid mosquitoes that over expresses antimicrobial peptides (AMP) such as Cecropin A and Defensin A under $\mathrm{Vg}$ promoters, after a blood meal in the haemolymph of female mosquitoes. The coordinated and cooperative action of these AMP has been shown to completely block the transmission of Plasmodium. ${ }^{19,20}$

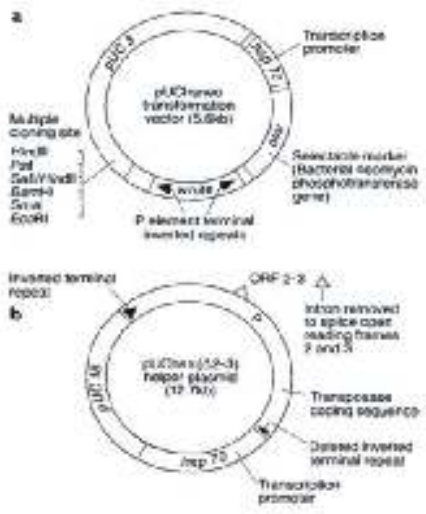

Figure 1. (a)The pUChsneo transformation vector carries an antibiotic resistance gene (neo) controlled by a Drosophila heat shock promoter (hsp70) and a multiple cloning site at which foreign DNA can be ligated between the inverted terminal repeats of the $\mathrm{P}$ transposable element. The repeats are linked by 500 bp of white locus DNA from Drosophila.(b) The pUChsTr(z52.-3) helper plasmid carries the entire $P$ transposase coding sequence which is transcribed under the control of the Drosophila hsp70 heat shock promoter. The sequence has been modified by invitro removal of an intron to overcome the germ line specificity observed in Drosophila. A deletion of one of the inverted terminal repeats of the helper prevents its integration into the target genome.

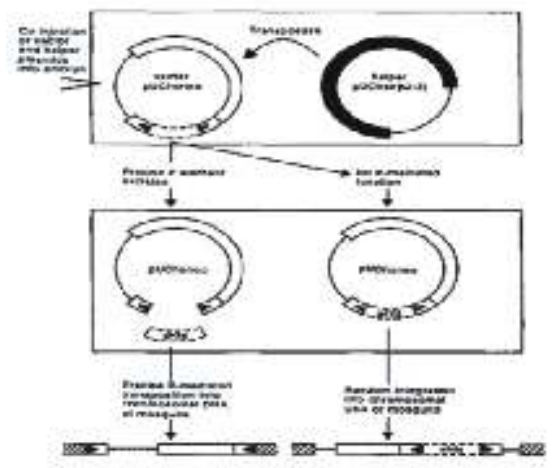

Figure 2. Co-injection of vector and helper plasmids into mosquito embryos. In a precise P-mediated transposition event, the transposase produced by the helper acts on the inverted terminal repeats to excise the white sequences precisely. This is followed by integration of all sequences 
within, and including, the inverted terminal repeats into the chromosomal DNA of the mosquitoes. If white sequences are detected in chromosomal DNA, it suggests that integration has occurred by some P-independent mechanism.

Riehle and his co-workers studied the genetically modified mosquitoes after feeding them malaria-infested blood, they noticed that the Plasmodium parasites did not infect a single study animal of the estimated 250 million people who contract malaria each year, 1 million mostly children do not survive. Ninety percent of the number of fatalities, which Riehle suspects to be underreported, occur in Sub-Saharan Africa. Each new malaria case starts with a bite from a vector a mosquito belonging to the genus Anopheles. About 25 species of Anopheles are significant vectors of the disease..$^{21,22}$ Only the female Anopheles mosquitoes feed on blood, which they need to produce eggs. When they bite an infected human or animal, they ingest the malaria parasite. Once the Plasmodium cells find themselves in the insect's midgut, they spring into action. They leave the insect's digestive tract by squeezing through the midgut lining.

The vast majority of Plasmodium cells do not survive this journey and are eliminated by the mosquito's immune cells. A tiny fraction of parasite cells, usually not more than a handful, make it and attach themselves on the outside of the midgut wall where they develop into brooding cells called oocysts. Within 10-12 days, thousands of new Plasmodium cells, socalled sporozoites, sprout inside the oocyst. After hatching from the oocyst, the sporozoites make their way into the insect's salivary glands where they lie in wait until the mosquito finds a victim for a blood meal. When the mosquito bites, some sporozoites are flushed into the victim's bloodstream. $^{24}$

The average mosquito transmits about 40 sporozoites when it bites," said Riehle, "but it takes only one to infect a human and make a new malaria victim."Several species of Plasmodium exist in different parts of the world, all of which are microscopically small single cell organisms that live in their hosts' red blood cells. ${ }^{25}$ Each time the parasites undergo a round of multiplication, their host cells burst and release the progeny into the bloodstream, causing the painful bouts of fever that malaria is known and feared for. The severity of the disease depends very largely on the species of the Plasmodium parasite the patient happens to contract. "Only two species of Plasmodium cause the dreaded relapses of the disease,". One of them, Plasmodium vivax, can lie dormant in the liver for 10 to 15 years, but now drugs have become available that target the parasites in the liver as well as those in the blood cells. That said, there are no effective or approved malaria vaccines. ${ }^{26} \mathrm{~A}$ few vaccine candidates have gone to clinical trials but they were shown to either be ineffective or provide only short-term protection. If an effective vaccine were to be developed, distribution would be a major problem, Riehle said. Researchers and health officials put higher hopes into eradication programs, which aim at the disease transmitting mosquitoes rather than the pathogens that cause it. ${ }^{27}$

"The eradication scenario requires three things: A gene that disrupts the development of the parasite inside the mosquito, a genetic technique to bring that gene into the mosquito genome and a mechanism that gives the modified mosquito an edge over the natural populations so they can displace them over time. "The third requirement is going to be the most difficult of the three to realize," he added, which is why his team decided to tackle the other two first. ${ }^{28}$ "It was known that the Akt enzyme is involved in the mosquito's growth rate and immune response, among other things," "So we went ahead with this genetic construct to see if we can ramp up Akt function and help the insects' immune system fight off the malaria parasite". ${ }^{29}$ The second rationale behind this approach was to use Akt signaling to stunt the mosquitoes' growth and cut down on its lifespan. "In the wild, a mosquito lives for an average of two weeks,". His research team discovered that mosquitoes carrying two copies.

Only the oldest mosquitoes are able to transmit the parasite. If we can reduce the lifespan of the mosquitoes, we can reduce the number of infections. Altered gene had lost their ability to act as malaria vectors altogether. "In that group of mosquitoes, not a single Plasmodium oocyte managed to form. At this point, the modified mosquitoes exist in a highly secured lab environment with no chance of escape. Once researchers find a way to replace wild mosquito populations with lab bred ones, breakthroughs like the one achieved by Riehle's group could pave the way toward a world in which malaria is all but history. ${ }^{30,31}$

\section{Mosquito Fitness}

The introduction of novel phenotypic traits in transgenic mosquitoes may induce a fitness cost that could impede the effectiveness of genetic drive mechanisms and thus the spread of transgenes in target field populations. ${ }^{32}$ Expression of marker proteins and effector molecules, particularly when expressed using ubiquitous promoters, may be detrimental to the mosquito. ${ }^{33}$ In addition, transgene insertion may interfere with gene expression of the mosquito strain because of the essentially random nature of genomic insertion. This so-called insertional mutagenesis may be naturally selected against or be overcome through selection of lines with higher fitness. ${ }^{34}$ However, the inability to control insertion site location using conventional vectors has led to the development of vectors that can provide a stable docking site for site-targeted 
transgene insertion. This, coupled with the use of suicide vectors, will increase safety and make transgene expression more predictable..$^{35}$ However, it will still be necessary to select, from a series of insertions with docking sites, the fit. Finally, the genetic make-up of transgenic lines and inbreeding have been posed as additional factors affecting the fitness of transgenic lines. ${ }^{32,36}$ This latter factor is probably not a serious concern and can probably be dealt with using a well-designed breeding scheme. These shortcomings may be overcome by circumventing the mosquito genome altogether and instead focus on genetic modification of symbiotic (midgut) bacteria as a means to deliver anti-pathogen products. ${ }^{37}$ Whatever approach is adopted in the coming years, compensation for fitness loss, preferably by conferring fitness advantages to transgenics that outweigh cost-benefit equilibria in susceptible wild-type mosquitoes will remain a critical challenge. ${ }^{38}$

The measurement of fitness in the laboratory with laboratory strains is at best inadequate and at worst misleading. The only meaningful measure of fitness will be when the GM mosquitoes are competed against wild mosquitoes in the field. As a concept to address this, Scott and others called for the development of consensus methodology in fitness studies based on stepwise progression from laboratory to semi-field settings, with competitiveness of GM mosquitoes as a key criterion. $^{39}$

\section{Genetic Drive}

On the premise that transgenic lines with acceptable fitness can be developed, the next challenge will be to drive the desirable attributes to fixation in field populations. In contrast with SIT campaigns, in which massive repeated releases of sterile males are practiced, the use of males able to drive a transgene into a target population may require the production and release of much smaller numbers, thereby gradually altering its susceptibility in favor of refractoriness. Reviews on genetic drive mechanisms and desirable attributes there of focus on the possible fitness costs associated with the drive mechanism itself, the rate at which introgression of transgenes in populations occurs, and the ability of the mechanism to drive large genetic constructs. ${ }^{34,36,40}$ Models have been developed to study these phenomena. These indicate that the complete linkage between genes delivering anti-pathogen traits, and the genetic drive mechanism deployed, besides stable and reliable levels of phenotypic expression of transgenes, is mandatory to accomplish adequate penetration of the target population and impact on disease transmission. If there is a fitness cost associated with a refractoriness effector gene, recombination between it and the driver will eventually lead to fixation of the driver alone without the effector gene, with no long-term impact on vector competence. A second major issue to take into account is the response of the target organism (i.e., the Plasmodium), to the anti-pathogen trait. This "trait" will be a protein that either prevents development of the parasite or kills it. The pressure on the parasite population will undoubtedly elicit a response given the time that the population replacement process may take. A mutational event that enables the parasite to negate the effects of the anti-pathogen trait will rapidly be selected for despite the ongoing gene driving process. Unfortunately, in contrast to the other two factors mentioned above, parasite response to selection pressure in the field is not a "researchable topic" and may only be studied under artificial laboratory conditions. Hopefully, targeting the Plasmodium population with parasiticidal compounds will not result in the development of resistance as occurred when the vector population was targeted with insecticidal compounds. ${ }^{41}$

\section{Transitional Challenges}

Given such accomplishments, a new array of challenges emerges. ${ }^{31}$ The complexity of these form the interface between the technological and the ELSI described below ${ }^{32}$. A stepwise approach has been proposed for fitness evaluations, and this model can be expanded to include components of the transitional change to bridge laboratory and field research. ${ }^{33,42}$ In furtherance to previous studies on transgenic insects, such as the pink bollworm, which were limited by tethering the insects and cutting off their wings, the endpoint of studies on GM mosquitoes will be their full evaluation in contained semi-field environments in a DEC setting. ${ }^{34}$ At least two of the four Grand Challenges in Global Health projects on genetic control of disease vectors (although these focus on Aedes rather than anopheline vectors) aim for this goal before 2010. Although this target is clear and restrictive in the sense that concerns related to open-field releases can be controlled, it does pose additional issues, particularly the choice of DEC setting. ${ }^{35,43}$

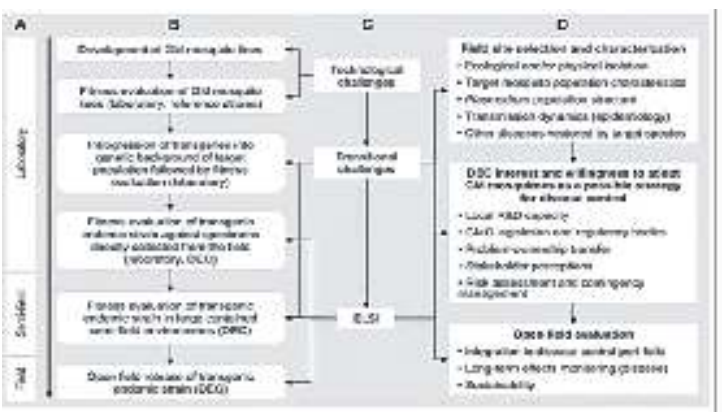

Figure 3. Staged progression of research toward field implementation of GM mosquitoes from laboratory to open field settings (A), the model proposed for fitness evaluation of 
transgenic mosquito lines (B; after Scott and others), and transitional and ELSI issues (C) affecting field site selection and open field releases $(\mathbf{D})$.

\section{Movement of mobile elements involves a DNA or RNA intermediate}

As research on mobile elements progressed, they were found to fall into two categories: (1) those that transpose directly as DNA and (2) those that transpose via an RNA intermediate transcribed from the mobile element by an RNA polymerase and then converted back into double-stranded DNA by a reverse transcriptase Mobile elements that transpose through a DNA intermediate are generally referred to as transposons. (As discussed below, this term has a more specific meaning in reference to bacterial mobile elements.) Mobile elements that transpose to new sites in the genome via an RNA intermediate are called retrotransposons because their movement is analogous to the infectious process of retroviruses. ${ }^{44}$ Indeed, retroviruses can be thought of as retrotransposons that evolved genes encoding viral coats, thus allowing them to transpose between cells. Both transposons and retrotransposons can be further classified based on their specific mechanism of transposition, as summarized in. We describe the structure and movement of the various types of mobile elements and then consider their likely role in evolution. ${ }^{45}$

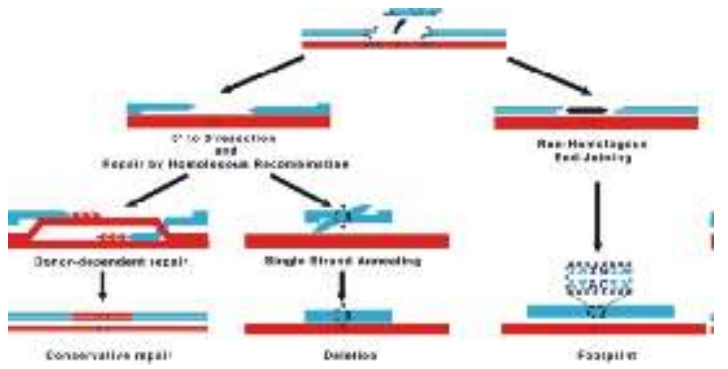

Figure 4.Transposon generates a double-strand break in the chromosome (light). In this example, the homologous chromosome (dark) is homozygous for the Tc insertion. The double-strand break is repaired by the cellular machinery using different possible pathways. Ends can be resected, resulting in $3^{\prime}$ single-stranded ends that can engage a recombination process

\section{REFERENCES}

1. Alphey L, Beard CB, Billingsley P, Coetze M, Crisanti A, et al. (2002) Malaria control with genetically manipulated insect vectors. Science 298: 119-121.

2. Beier JC (2008) Malaria control in the highlands of Burundi: An important success story. Am J Trop Med Hyg 79: 1-2.

3. Benedict M, D'Abbs P, Dobson S, Gottlieb M, Harrington L, et al.(2008) Guidance for contained field trials of vector mosquitoes engineered to contain a gene drive system:
Recomm-endations of a scientific working group. Vector Borne Zoonotic Dis 8: 127-166.

4. Bruce-Chwat LJ, de Zulueta J (1980) The rise and fall of malaria in Europe: A historicoepidemiological study. Oxford, New York: Oxford University Press. 256 p.

5. De Lara Capurro M, Coleman J, Beerntsen BT, Myles KM, Olson KE, et al. (2000) Virusexpressed, recombinant singlechain antibody blocks sporozoite infection of salivary glands in Plasmodium gallinaceum-infected Aedes aegypti. Am J Trop Med Hyg 62: 427-433.

6. Hay SI, Guerra CA, Tatum AJ, Atkinson PM, Snow RW (2005) Urbanization, malaria transmission and disease burden in Africa. Nat Rev Microbiol 3: 81-90.

7. Humphreys M (2001) Malaria: Poverty, race and public health in the United States. Baltimore: Johns Hopkins University Press. 208 p.

8. Ito J, Ghosh A, Moreira LA, Wimmer EA, Jacobs-Lorena M (2002) Transgenic anopheline mosquitoes impaired in transmission of a malaria parasite. Nature 417: 452-455.

9. Lavery JV, Harrington LC, Scott TW (2008) Ethical, social and cultural considerations for site selection for research with genetically modified mosquitoes. Am J Trop Med Hyg 79: 312318.

10. Marshall JM (2009) The effect of gene drive on containment of transgenic mosquitoes. J Theor Biol. 258(2):250-265.

11. Ridley RG (2002) Medical need, scientific opportunity and the drive for antimalarial drugs. Nature 415: 686-693.

12. Riehle MM, Markianos K, Niare O, Xu J, Li J, et al. (2006) Natural malaria infection in Anopheles gambiae is regulated by a single genomic control region. Science 312:577-579.

13. Rong YS, Golic KG (2003) The homologous cromosome is an effective template for the repair of mitotic DNA double-strand breaks in Drosophila. Genetics 165: 1831-1842.

14. Snow RW, Guerra CA, Noor AM, Myint HY, Hay SI (2005) The global distribution of clinical episodes of Plasmodium falciparum malaria. Nature 434: 214-217.

15. Taylor C, Touré YT, Carnahan J, Norris DE, Dolo G, et al. (2001) Gene flow among populations of the Malaria vector Anopholes gambiae in Mali, West Africa. Genetics 157: 743-750.

16. Todryk SM, Hill AVS (2007) Malaria vaccines: The stage we are at. Nat Rev Microbiol 5: 487-490.

17. Waterhouse RM, Kriventseva EV, Meister S, Xi Z, Alvarez KS, et al. (2007) Evolutionary dynamics of immune-related genes and pathways in disease-vector mosquitoes. Science 316: 17381743.

18. Yamey G (2004) Roll Back Malaria: A failing global health campaign. BMJ 328: 1086-1087.

19. Jasinskiene N, Coates CJ, Benedict MQ, Cornel AJ, Rafferty CS, et al. (1998) Stable transformation of the yellow fever mosquito, Aedes aegypti, with the Hermes element from the 
housefly. Proc Natl Acad Sci U S A 95: 3743-3747.

20. Franz AW, Sanchez-Vargas I, Adelman ZN, Blair CD, Beaty BJ, et al. (2006) Engineering RNA interference-based resistance to dengue virus type 2 in genetically modified Aedes aegypti. Proc Natl Acad Sci U S A 103: 4198-4203.

21. Ito J, Ghosh A, Moreira LA, Wimmer EA (2002) Transgenic Anopheline Mosquitoes Impaired in Transmission of a Malaria Parasite. Nature, 417: 452-455.

22. Sperança MA, Capurro ML (2007) Perspectives in the control of infectious diseases by transgenic mosquitoes in the postgenomic eraa review. Mem Inst Oswaldo Cruz 102: 425-433.

23. Walker T, Johnson PH, Moreira LA, Iturbe-Ormaetxe I, Frentiu FD, et al. (2011) The wMel Wolbachia strain blocks dengue and invades caged Aedes aegypti populations. Nature 476: 450-453.

24. Kokoza V, Ahmed A, Wimmer EA, Raikhel AS (2001) Efficient Transformation of the Yellow Fever Mosquito Aedes Aegypti Using the piggyBac Transposable Element Vector pBac[3xP3-EGFPAfm].Insect Biochem Mol Biol 3:1137-1143.

25. Phuc HK, Andreasen MH, Burton RS, Vass C, Epton MJ, et al. (2007) Late-acting dominant lethal genetic systems and mosquito control. BMC Biol 5: 11.

26. Fu G, Lees RS, Nimmo D, Aw D, Jin L, et al. (2010) Femalespecific flightless phenotype for mosquito control. Proc Natl Acad Sci U S A 107: 4550-4554.

27. Kokoza V, Ahmed A, Woon Shin S, Okafor N, Zou Z, et al. (2010) Blocking of Plasmodium transmission by cooperative action of Cecropin A and Defensin A in transgenic Aedes aegypti mosquitoes. Proc Natl Acad Sci U S A 107: 8111-8116.

28. Windbichler N, Papathanos PA, Crisanti A (2008) Targeting the $\mathrm{X}$ Chromosome during Spermatogenesis Induces Y Chromosome Transmission Ratio Distortion and Early Dominant Embryo Lethality in Anopheles Gambiae. PLoS Genet 4:1-9.

29. World Health Organization. World Malaria Report 2013.

30. Gabrieli P, Smidler A, Catteruccia F (2014) Engineering the control of mosquito-borne infectious diseases. Genome Biol 15: 535 .

31. Klein TA, Windbichler N, Deredec A, Burt A, Benedict MQ (2012) Infertility resulting from transgenic I-PpoI male Anopheles gambiae in large cage trials. Pathog Glob Health 106: 20-31.

32. Marrelli MT, Moreira CK, Kelly D, Alphey L, Jacobs-Lorena M. (2006) Mosquito transgenesis: what is the fitness cost? Trends Parasitol.;22:197202.

33. Moreira LA, Wang J, Collins FH, Jacobs-Lorena M. (2004) Fitness of anopheline mosquitoes expressing transgenes that inhibit Plasmodium development. Genetics.;166:1337-1341.

34. Lyman RF, Lawrence F, Nuzhdin SV, Mackay TF. (1996) Effects of single P-element insertions on bristle number and viability in Drosophila melanogaster. Genetics. ;143: 277-292.
35. Horn C, Handler AM. (2005) Site-specific genomic targeting in Drosophila. Proc Natl Acad Sci USA.;102:12483-12488.

36. Catteruccia F, Godfray HC, Crisanti A. (2003) Impact of genetic manipulation on the fitness of Anopheles stephensimosquitoes. Science;299:1225-1227.

37. Riehle MA, Jacobs-Lorena M. (2005) Using bacteria to express and display anti-parasite molecules in mosquitoes: current and future strategies. Insect Biochem Mol Biol.;35:699-707.

38. Ferguson H, Gandon S, Mackinnon M, Read A. (2006) Malaria parasite virulence in mosquitoes and its implications for the introduction and efficacy of GMM malaria control programmes. In: Boëte C, editor. Genetically Modified Mosquitoes for Malaria Control. Georgetown: Eurekah/Landes Bioscience; 103-116.

39. Scott TW, Rasgon JL, Black WC IV, Gould F. (2006) Fitness studies: developing a consensus methodology. In: Knols BGJ, Louis C, editors. Bridging Laboratory and Field Research for Genetic Control of Disease Vectors.Dordrecht: Springer; 171-181.

40. James AA. (2005) Gene drive systems in mosquitoes: rules of the road. Trends Parasitol.;21:64-67.

41. Braig HR, Yan G. (2001) The spread of genetic constructs in natural insect populations. In: Letourneau DK, Burrows BE, editors. Genetically Engineered Organisms: Assessing Environmental and Human Health Effects. Boca Raton: CRC Press; 251-314.

42. Rasgon JL, Gould F. (2005) Transposable element insertion location bias and the dynamics of gene drive in mosquito populations. Insect Mol Biol;14:493-500.

43. Curtis CF. (2006) Models to investigate some issues regarding the feasibility of driving refractoriness genes into mosquito vector populations. In: Knols BGJ, Louis C, editors. Bridging Laboratory and Field Research for Genetic Control of Disease Vectors. Dordrecht: Springer; 199-202.

44. Curtis CF, Coleman PG, Kelly DW, Campbell-Lendrum DH. (2006) Advantages and limitations of transgenic vector control: sterile males versus gene drivers. In: Boëte $C$, editor. Georgetown: Eurekah/Landes Bioscience; Genetically Modified Mosquitoes for Malaria Control. 60-78.

45. Jacobs-Lorena M, James AA. (2003) Genetic Modification of Insects of Medical Importance: Past, Present and Future. Geneva: World Health Organization. 